\title{
Regional volumes and spatial volumetric distribution of gray matter in the gender dysphoric brain
}

\author{
Elseline Hoekzema ${ }^{a, *, 1}$, Sebastian E.E. Schagen ${ }^{c, e, 1}$, \\ Baudewijntje P.C. Kreukels ${ }^{\mathrm{b}}$, Dick J. Veltman ${ }^{\mathrm{d}}$, \\ Peggy T. Cohen-Kettenis ${ }^{\mathrm{b}}$, Henriette Delemarre-van de Waal ${ }^{\mathrm{e}, 2}$, \\ Julie Bakker ${ }^{a, f}$
}

\footnotetext{
a Neuroendocrinology Bakker Group, Netherlands Institute for Neuroscience, Amsterdam, The Netherlands

${ }^{b}$ Center of Expertise on Gender Dysphoria, Department of Medical Psychology, Neuroscience Campus Amsterdam, VU University Medical Center, Amsterdam, The Netherlands

c Department of Pediatric Endocrinology, Neuroscience Campus Amsterdam, VU University Medical Center, Amsterdam, The Netherlands

d Department of Psychiatry, Neuroscience Campus Amsterdam, VU University Medical Center, Amsterdam, The Netherlands

e Department of Pediatrics, Leiden University Medical Center, Leiden The Netherlands

f Centre Interdisciplinaire de Géneoprotéomique Appliquée, Université Liège, Liège, Belgium
}

Received 12 September 2014; received in revised form 29 December 2014; accepted 21 January 2015

\section{KEYWORDS \\ MRI; \\ Transsexual; \\ Transsexualism; \\ Gender identity \\ disorder; \\ Voxel-based \\ morphometry; \\ Sex differences}

\begin{abstract}
The sexual differentiation of the brain is primarily driven by gonadal hormones during fetal development. Leading theories on the etiology of gender dysphoria (GD) involve deviations herein. To examine whether there are signs of a sex-atypical brain development in GD, we quantified regional neural gray matter (GM) volumes in 55 female-to-male and 38 male-to-female adolescents, 44 boys and 52 girls without GD and applied both univariate and multivariate analyses. In girls, more GM volume was observed in the left superior medial frontal cortex, while boys had more volume in the bilateral superior posterior hemispheres of the cerebellum and the hypothalamus. Regarding the GD groups, at whole-brain level they differed only from individuals sharing their gender identity but not from their natal sex. Accordingly, using multivariate pattern recognition analyses, the GD groups could more accurately be automatically discriminated from individuals sharing their gender identity than those sharing their natal sex based on spatially distributed GM patterns. However, region of interest analyses indicated less GM volume in the right cerebellum and more volume in the medial frontal cortex in female-to-males in comparison to girls without GD, while male-to-females had less volume in
\end{abstract}

\footnotetext{
* Corresponding author. Tel.: +31 0204444943.

E-mail address: e.hoekzema@nin.knaw.nl (E. Hoekzema).

1 Shared first authorship.

2 Deceased.
} 
the bilateral cerebellum and hypothalamus than natal boys. Deviations from the natal sex within sexually dimorphic structures were also observed in the untreated subsamples. Our findings thus indicate that GM distribution and regional volumes in GD adolescents are largely in accordance with their respective natal sex. However, there are subtle deviations from the natal sex in sexually dimorphic structures, which can represent signs of a partial sex-atypical differentiation of the brain.

(c) 2015 Elsevier Ltd. All rights reserved.

\section{Introduction}

During a critical period of fetal and neonatal development, gonadal hormones are thought to primarily drive the sexual differentiation of the brain, sculpting brain morphology into a gender-typical configuration. In genetic males, the testes secrete high levels of testosterone during fetal life, rendering serum levels in the adult male range. Testosterone can be converted to 17- $\beta$-estradiol by the enzyme aromatase, and animal studies have demonstrated that pre- and perinatal estradiol acts as the principal regulator of the sexual differentiation of the rodent brain (Bakker and Baum, 2008; McCarthy, 2008). Cerebral masculinization is precluded in genetic females by the estrogen-binding activity of alphafetoprotein, a plasma protein secreted by the fetal liver in high concentrations (Bakker et al., 2006; Bakker and Baum, 2008). Manipulating the exposure to sex steroid hormones during a critical period of prenatal and early postnatal life substantially perturbs the sex-specific differentiation of the brain (Bakker et al., 2006; Bakker and Baum, 2008).

In humans, pre- and perinatal neuroendocrine factors are also thought to primarily regulate the sexual differentiation of the brain. Interestingly, individuals $(46, \mathrm{XY})$ with complete androgen insensitivity syndrome, who have nonfunctional androgen receptor proteins due to a mutation in the AR gene, are born phenotypically female, are sexually attracted to men and have a female gender identity (Wisniewski et al., 2000; Hines et al., 2003), suggesting that in humans masculinization may be directed by testosterone rather than testosterone-derived estradiol. Although the sexual differentiation of the brain is believed to primarily take place during prenatal development, circulating hormones during adolescence exert activational effects and further potentiate neural sexual dimorphisms. In addition to gonadal hormone effects, direct effects of genes residing on the sex chromosomes may also contribute to the sexual differentiation of the brain (Arnold, 2004; Carruth et al., 2002).

In gender dysphoria (GD) there is a persistent incongruence between a person's natal sex and the experienced gender identity. Leading theories on the etiology of this condition involve a sex-atypical cerebral programming that diverges from the sexual differentiation of the rest of the body, postulated to reflect the organizational effects of altered levels of sex steroid hormones during a specific period of fetal development (Bao and Swaab, 2011; CohenKettenis and Gooren, 1999; Savic et al., 2010; Swaab, 2007). Some twin studies suggest a role for genetic factors in the development of GD, potentially involving polymorphisms in genes encoding elements of the sex steroid signaling or metabolic pathways (Heylens et al., 2012).
Postmortem studies have observed sex-atypical volumes and neuronal numbers in hypothalamic nuclei in the brains of individuals with GD (Garcia-Falgueras and Swaab, 2008; Kruijver et al., 2000; Zhou et al., 1995). Brain structure in individuals with GD has also been investigated in vivo. MRI studies examining white matter tracts using diffusion tensor imaging have shown deviations from the natal sex in white matter microstructure in both female-to-males (FM) and male-to-females (MF) (Rametti et al., 2011a,b). A few neuroimaging studies have investigated gray matter (GM) in individuals with GD, observing both regional volumetric properties in line with the natal sex and others in line with the gender identity of the GD groups (Luders et al., 2009b, 2012; Savic and Arver, 2011; Zubiaurre-Elorza et al., 2012; Simon et al., 2013). A recent study by Zubiaurre-Elorza et al. (2012) was the first to examine GM in FM (ZubiaurreElorza et al., 2012). Interestingly, they observed signs of subcortical masculinization in female-to-male adults and of cortical feminization in male-to-female adults, providing the first insights into the developmental processes underlying GD in both sexes.

In our study, we wanted to examine brain anatomy in individuals with GD during adolescence, when puberty-related sex steroid hormones are driving the further 'activational' sexual differentiation of the brain rather than in adulthood, when both organizational and activational steroid hormone effects have already sculpted the brain into a sex-specific configuration. Therefore, we acquired highresolution anatomical brain $M R I$ scans of 55 female-to-male adolescents and 38 male-to-female (MF) adolescents with GD. In addition, to allow comparisons with individuals sharing either their gender identity or their natal sex, we also scanned 44 boys and 52 girls without GD going through adolescence.

First, we aimed to define sexually dimorphic structures in the adolescent brain by comparing GM volume in boys and girls without GD. As the principal aim of our study was to examine whether signs of sex-atypical cerebral programming are present in the brains of GD adolescents, we subsequently compared their regional GM volumes to individuals sharing either their gender identity or their natal sex. To ensure that the deviations from the natal sex did not result from hormone therapy, we repeated these analyses in the subsamples of GD participants who had never been exposed to any form of hormonal treatment.

Finally, considering the recent development of sophisticated machine learning algorithms for MRI analyses, we also wanted to explore the data beyond the classical mass-univariate statistical approach. Especially relevant for the study of individuals with GD is that multivariate pattern recognition approaches provide a tool to quantify the 
predictive value of volumetric GM patterns for group discrimination. Therefore, the final aim of our study was to examine these data using a multivariate approach and assess the extent to which individuals with GD can be discriminated from either of the control groups based on the spatially distributed patterns of GM volume in the brain.

\section{Materials and methods}

\subsection{Subjects}

Subjects for this study were recruited by the Center of Expertise on Gender Dysphoria of the VU University Medical Center in Amsterdam as part of a larger research project investigating brain development, brain functioning, growth and metabolic aspects in the clinical management of adolescents with GD (Trial registration number ISRCTN 81574253 (http://www.controlled-trials.com/isrctn/)). All adolescents with Gender Identity Disorder were evaluated according to the standard protocol at the clinic using the criteria described in the Diagnostic and Statistical Manual of Mental Disorders-IV-TR (DSM-IV-TR) (American Psychiatric Association, 2000). Please note that, in this version of the DSM, 'Gender Identity Disorder' was still the official name of the diagnosis. Therefore, only in the context of describing the diagnostic procedure we will use this term rather than the more recent 'Gender Dysphoria'. When participants were referred to the clinic during childhood, the child and parents participated in several sessions with a mental health care professional specialized in children and adolescents with Gender Identity Disorder. During these sessions, the aim was to determine whether the criteria for a Gender Identity Disorder diagnosis in children according to DSM-IVTR were met, to evaluate the cognitive, psychological and social functioning of the child and functioning of the family, and, if indicated, to offer or refer for psychological treatment or counseling. The core criteria for a DSM-IV-TR Gender Identity Disorder diagnosis are a strong and persistent crossgender identification and a persistent discomfort with his or her sex or sense of inappropriateness in the gender role of that sex. As a result, the child should experience a significant amount of distress or impairment in important areas of their functioning. In adolescence, the individuals (re-)applied to the clinic, where they entered the diagnostic procedure for adolescents. This implied an evaluation of the presence of Gender Identity Disorder in adolescents/adults and other psychopathology according to DSM-IV-TR criteria.

The current analyses were performed several years after data acquisition, which allowed us to ascertain that the sample consisted only of individuals with persistent GD. In our study, we included only participants who had been followed at the center for many years and who had indeed completed or were in the last phase of cross-sex hormone treatment at the time of the analyses rather than relying on participants' declaration of intending to undergo progressive treatment steps to change their body to fit their gender identity. Two individuals had to be removed from the analyses as it became clear at some point after the data acquisition that their GD had not persisted into adulthood. Our final sample consisted of 54 female-to-male adolescents, 37 male-to-female adolescents, 44 natal male and 52 natal female adolescents without GD.

After receiving the diagnosis of Gender Identity Disorder in adolescence and meeting some additional criteria to be eligible for treatment (Kreukels and Cohen-Kettenis, 2011), they were referred for hormonal treatment. The standard for treatment in the clinic follows the Standards of Care of the World Professional Association for Transgender Health (WPATH, Coleman et al., 2011), a professional organization in the field of transgender care, and the Clinical Practice Guidelines of the Endocrine Society published in 2009 (Hembree et al., 2009), comprising puberty suppression with gonadotrophin releasing hormone $(\mathrm{GnRH})$ analogues once adolescents start to display the first physical and endocrine changes of puberty (Tanner stage 2-3), followed by cross-sex hormone therapy after the age of 16. From 18 years of age onward, individuals with GD can be referred for sex reassignment surgery. After gonadectomy, GnRH analogues are no longer provided. Treatment with cross-sex hormones is continued after sex reassignment surgery. For additional information regarding the standards for diagnostics and treatment of adolescents with GD, see (Hembree et al., 2009; Kreukels and Cohen-Kettenis, 2011). Please see Table 1 for demographic and clinical details of the sample.

Depending on the age of the participant, intelligence was estimated with the Wechsler Intelligence Scale for Children (WISC-III) (Wechsler, 1991) or the Wechsler Adult Intelligence Scale (WAIS-III) (Wechsler, 1997). All participants also underwent a physical examination by a clinician to determine height, weight, blood pressure and puberty stage according to Tanner (1962). Tanner staging, which defines the progress of puberty based on the development of secondary sex characteristics, is considered the gold standard for puberty measurement when performed by a trained clinician (Dorn, 2006).

The FM were all gynephilic, whereas the MF were androphilic (i.e. sexually attracted to partners of their natal sex), with the exception of one of the MF who reported to be indifferent with regard to the sex of the partner. Repeating the analyses without this subject did not affect our results.

All GD participants had started to experience GD symptoms in childhood and met criteria for 'early-onset' gender dysphoria. Rather than assessing whether symptoms had commenced before puberty by retrospective questionnaires, we were able to ensure that our participants' GD had started in childhood as they had enrolled in our clinic at an early age and had been clinically evaluated for many years.

No participants with any known physical inter-sex conditions, neurological disorders, cerebral damage or major medical conditions were included in this study. To assess psychological functioning in all groups, the Dutch translation of the Child Behavior Checklist (Achenbach and Edelbrock, 1983; Verhulst et al., 1996) was used. In case of scores in the clinical range indicating problem behavior, participants were not included in the study.

The study was approved by the Ethical Review Board of the VU Medical Center. Written informed consent was obtained from the subjects and from the parents or guardians of the child participants prior to their participation in the study. 
Table 1 Clinical and demographic data. GnRH: GD individuals undergoing treatment with GnRH analogues but not cross-sex hormones at the time of the MRI acquisition. Cross-sex: GD individuals undergoing cros-sex hormone therapy.

\begin{tabular}{|c|c|c|c|c|c|c|c|}
\hline Group (N) & $\begin{array}{l}\text { Treatment } \\
\text { stage }(\mathrm{N})\end{array}$ & $\begin{array}{l}\text { Age }(M \pm S D) \\
\text { (range) }\end{array}$ & $\begin{array}{l}\text { Tanner }(M \pm S D) \\
\text { (range) }\end{array}$ & $\mathrm{IQ}(\mathrm{M} \pm \mathrm{SD})$ (range) & $\begin{array}{l}\text { Duration } G n R H \\
\text { (days) }(M \pm S D) \\
\text { (range) }\end{array}$ & $\begin{array}{l}\text { Duration cross-sex } \\
\text { (days) }(\mathrm{M} \pm \mathrm{SD}) \\
\text { (range) }\end{array}$ & $\begin{array}{l}\text { Cumulative dosis } \\
\text { cross-sex }^{* *}(\mathrm{mg}) \\
(\mathrm{M} \pm \mathrm{SD}) \text { (range) }\end{array}$ \\
\hline M (44) & & $16.42 \pm 2.75(10.54)$ & $4.35 \pm 1.09(3)$ & $110.5 \pm 19.88(85)$ & - & - & - \\
\hline$F(52)$ & & $16.29 \pm 2.96(11.49)$ & $4.55 \pm 0.76(3)$ & $105.19 \pm 16.32(60)$ & - & - & - \\
\hline \multirow[t]{4}{*}{ FM (54) } & $\begin{array}{l}\text { Untreated } \\
\text { (17) }\end{array}$ & $15.20 \pm 2.76(9.26)$ & $4.12 \pm 1.15$ & $101.82 \pm 14.65(50)$ & - & - & - \\
\hline & GnRH (16) & $15.88 \pm 1.54(4.27)$ & $3.62 \pm 1.41(4)$ & $93.31 \pm 14.71(49)$ & $611.31 \pm 435.22(1435)$ & - & - \\
\hline & $\begin{array}{l}\text { Cross-sex } \\
(21)^{*}\end{array}$ & $19.11 \pm 1.29(4.90)$ & $2.86 \pm 1.88$ & $105.29 \pm 17.27(51)$ & $1595.67 \pm 785.92(3328)$ & $1025.25 \pm 436.21(1586)$ & $9270.38 \pm 9616.43(39,082)$ \\
\hline & $\begin{array}{l}\text { Complete } \\
\text { sample }\end{array}$ & $16.92 \pm 2.84(10.24)$ & $3.47 \pm 1.61(4)$ & $100.65 \pm 16.24(58)$ & $1170.00 \pm 816.39(3796)$ & $1025.25 \pm 436.21(1586)$ & $9270.38 \pm 9616.43(39,082)$ \\
\hline \multirow[t]{4}{*}{ MF (37) } & $\begin{array}{l}\text { Untreated } \\
\text { (11) }\end{array}$ & $13.77 \pm 2.42(7.99)$ & $3.45 \pm 1.13$ & $112.45 \pm 22.71(70)$ & - & - & - \\
\hline & GnRH (14) & $15.29 \pm 0.73(2.22)$ & $3.71 \pm 0.99(2)$ & $93.86 \pm 10.75(38)$ & $692 \pm 247.65(952)$ & - & - \\
\hline & $\begin{array}{l}\text { Cross-sex } \\
(12)^{*}\end{array}$ & $19.04 \pm 2.17(7.11)$ & $4.17 \pm 0.84(2)$ & $107.83 \pm 18.97(58)$ & $1619.75 \pm 398.01(1232)$ & $911.76 \pm 497.02(1352)$ & $1658.17 \pm 1133.68$ (3867) \\
\hline & $\begin{array}{l}\text { Complete } \\
\text { sample }\end{array}$ & $16.05 \pm 2.84(11)$ & $3.78 \pm 01.00$ & $103.92 \pm 19.02(70)$ & $1137.32 \pm 571.89(2141)$ & $911.76 \pm 497.02(1352)$ & $1658.17 \pm 1133.68(3867)$ \\
\hline
\end{tabular}

\footnotetext{
* Four of the MF and nine of the FM had undergone sex reassignment surgery.

FM: testosterone (Sustanon), and MF: estradiol (Progynova/Meno-implant).
} 


\subsection{MRI acquisitions}

The MRI images were acquired with a 3 T Philips Intera scanner, equipped with a SENSE 6-channel head coil. To obtain a high-resolution anatomical brain image, a T1-weighted pulse sequence was applied using the following acquisition parameters: $\mathrm{TR}=9 \mathrm{~ms}, \mathrm{TE}=3.5 \mathrm{~ms}$, matrix size $=256 \times 256$, voxel size $=1 \times 1 \times 1 \mathrm{~mm}$, and number of slices $=170$.

\subsection{Univariate analyses}

MRI image processing and statistical analyses were conducted with Statistical Parametric Mapping software (SPM8; www. fil.ion.ucl.ac.uk-spm), implemented in Matlab (version 7.8; www. mathworks.com).

Regional GM volume was quantified using a voxelbased morphometric approach, a fully automated technique designed to evaluate local volumetric properties of brain anatomy (Ashburner and Friston, 2000). We used the analysis pipeline provided in the Gaser vbm8 toolbox (http://dbm.neuro.uni-jena.de/vbm/). With this toolbox, the anatomical images were segmented into different tissue classes using an adaptive maximum a posteriori approach corrected for field inhomogeneities and brought into standard space using affine and non-linear normalization.

Since we worked with a sample of adolescent participants, we created a customized sample-specific DARTEL template for our study. First, to obtain suitable initial agematched tissue probability maps for import into DARTEL, we generated a customized age-matched template and tissue priors using the TOM8 toolbox (https://irc.cchmc.org/ software/tom.php) and used these in an affine registration. The affine registered gray matter and white matter segments were then used to create a sample-specific DARTEL template, which was then implemented in a DARTEL normalization to warp our data into MNI space. To preserve original tissue volumes and allow comparisons of volumes rather than GM concentrations, we used the GM segments that had been multiplied by the Jacobian determinants extracted from the normalization step. Finally, an $8 \mathrm{~mm}$ full width at half maximum smoothing kernel was applied to the data. An absolute threshold of 0.2 was applied to the analyses, and total GM volume of the brain was included as a nuisance covariate.

As there was a significant difference in IQ between the groups $(M \pm S D, M: 110.50 \pm 19.88, F: 105.19 \pm 16.32$, MF: $103.92 \pm 19.02, \mathrm{FM}: 100.66 \pm 16.24 . F=2.54, p=0.058)$, IQ was included in the between-group comparisons. We observed no correlations between regional GM volumes and IQ within the groups. When subgroups of the total sample were compared causing differences in age between the groups to surface, then age was also included as a covariate of no interest.

The whole-brain results are reported at a threshold of $p<0.05$ FWE-corrected and an extent threshold of $k>10$ voxels. Moreover, to examine whether more subtle deviations from the natal sex could be observed within sexually dimorphic brain structures, regions of interest (ROIs) were created representing these regions. ROI analyses were performed in Marsbar (http: / / marsbar.sourceforge.net/).

Considering our hypothesis that individuals with GD have undergone a sex-atypical differentiation of the brain that diverges from their natal sex in the direction of the cerebral developmental processes typical for the other sex, the ROI of larger female volume was applied to the contrasts ' $F>M F$ ' and ' $M F>M$ ', and the ROls of larger male volume were applied to the contrasts 'MF $>\mathrm{F}$ ' and ' $\mathrm{FM}>\mathrm{F}$ '. The $p$ values for the ROI results are Bonferroni-corrected for the number of ROls.

In addition to analyses involving the complete sample, we also separately compared the GD individuals who had never been exposed to any form of hormonal therapy (excluding both the individuals whose puberty was suppressed by means of $\mathrm{GnRH}$ analogues and the GD participants who had initiated cross-sex hormone therapy at the time of the MRI acquisition) to age-matched control samples of both sexes. To minimize the contribution of puberty-related endogenous sex steroid hormones and focus primarily on organizational effects, the stage of pubertal development (as an index of the degree of puberty-related endogenous sex steroid hormones the individual had been exposed to until the time of the MRI acquisition) was included as a nuisance covariate in these analyses.

Furthermore, we also compared untreated FM and MF to the GD participants who had already initiated cross-sex hormone therapy at the time of MRI acquisitions.

\subsection{Multivariate analyses}

In addition to the above-described mass-univariate statistical approach, we also performed a pattern recognition analysis. This is a method that stems from machine learning, and uses a multivariate approach to investigate spatially distributed information in MRI data - which is intrinsically multivariate - and hereby allows modeling signal patterns in the images. In our study, we used the analysis pipeline for pattern recognition analyses provided by PRoNTo (http://www.mlnl.cs.ucl.ac.uk/pronto). This pipeline can be used to automatically search for regularities in the data and train a classifier function that models the relation between spatial signal patterns and experimental factors (in our case, group) based on a training dataset (Schrouff et al., 2013). This classifier can then be used to predict the group a new image belongs to using the spatial distribution of the signal within the image, and compute the accuracy with which groups can be discriminated from one another based on whole-brain spatial signal patterns. We selected the binary support vector machine (SVM) and a leave-oneout cross-validation strategy. This cross-validation strategy computes the accuracy of classifiers by leaving one subject out at a time and predicting the group label of this subject based on a training dataset including all remaining subjects, which is repeated for each subject. The results of all these runs are then used to compute the accuracy of the discriminant function. No covariates were included in the multivariate analyses. First we trained and tested a linear SVM pattern classifier for the natal boys and girls without $G D$, to assess the accuracy with which they could be discriminated from one another based on the distribution of GM volume. Then, we repeated these analyses for the GD groups and individuals sharing either their gender identity or their natal sex. These multivariate analyses were also repeated including only those MF and FM who had never been exposed 
Table 2 GM group comparisons. Full results of the whole-brain comparisons between adolescent boys and girls and between the GD groups and adolescents sharing their gender identity, reported at a whole-brain statistical threshold of $p<0.05 \mathrm{FWE}$-corrected and an extent threshold of 10 voxel.

\begin{tabular}{|c|c|c|c|c|c|c|c|}
\hline \multirow[t]{2}{*}{ Comparison } & \multirow[t]{2}{*}{ Brain region } & \multicolumn{3}{|c|}{ MNI coordinates } & \multirow[t]{2}{*}{ Cluster size $\left(\mathrm{mm}^{3}\right)$} & \multirow[t]{2}{*}{$T$} & \multirow[t]{2}{*}{$P$ (FWE-corrected) } \\
\hline & & $x$ & $y$ & $z$ & & & \\
\hline \multicolumn{8}{|c|}{ Comparisons boys and girls without GD } \\
\hline \multirow[t]{5}{*}{$M>F$} & L Cerebellum & -42 & -76 & -20 & 591 & 5.38 & 0.002 \\
\hline & R Cerebellum & 41 & -46 & -29 & 2714 & 5.71 & $<0.001$ \\
\hline & & 45 & -73 & -20 & & 5.45 & 0.002 \\
\hline & & 32 & -37 & -26 & & 5.32 & 0.003 \\
\hline & Hypothalamus & 6 & 2 & -2 & 243 & 5.38 & 0.001 \\
\hline \multirow[t]{2}{*}{$\mathrm{F}>\mathrm{M}$} & $\begin{array}{l}\text { L Superior Medial } \\
\text { Frontal Cortex }\end{array}$ & -8 & 63 & 22 & 206 & 5.13 & 0.006 \\
\hline & & -8 & 69 & 15 & & 5.02 & 0.009 \\
\hline \multicolumn{8}{|c|}{ Comparisons GD to groups sharing gender identity } \\
\hline \multirow[t]{2}{*}{$F>M F$} & $\begin{array}{l}\text { R Superior Medial } \\
\text { Frontal/R Superior } \\
\text { Frontal Cortex }\end{array}$ & 18 & 57 & 33 & 164 & 6.01 & $<0.001$ \\
\hline & & 6 & 59 & 37 & & 5.01 & 0.006 \\
\hline$M F>F$ & L Cerebellum & -15 & -48 & -59 & 47 & 4.80 & 0.022 \\
\hline$M>F M$ & $\begin{array}{l}\text { L Cerebellum/L } \\
\text { Fusiform }\end{array}$ & -42 & -78 & -18 & 54 & 4.86 & 0.017 \\
\hline$F M>M$ & - & & & & & & \\
\hline \multicolumn{8}{|c|}{ Comparisons GD to groups sharing natal sex } \\
\hline$M>M F$ & - & & & & & & \\
\hline$M F>M$ & - & & & & & & \\
\hline$F>F M$ & - & & & & & & \\
\hline$F M>F$ & - & & & & & & \\
\hline
\end{tabular}

to any hormonal treatment and their age-matched controls. Balanced accuracy values, which represent the fraction of the subjects for whom a correct class label was predicted using the trained classifier, are provided for each of the comparisons. Permutation tests were used to examine whether the classifier performed above chance level.

\section{Results}

\subsection{Univariate results}

\subsubsection{Complete sample}

To identify regions of sexual dimorphism in the adolescent brain, we first compared regional GM volumes (corrected for total GM volume) between girls and boys without GD. These whole-brain analyses (thresholded at $p<0.05$ FWEcorrected and an extent threshold of $k>10$ voxels) rendered three localized clusters of larger GM volumes in boys and one cluster where girls had more GM volume (see Table 2 ). More specifically, in girls, enlarged volume was observed in a section of the left frontal cortex extending from the superior part of the medial frontal cortex to the superior frontal cortex. For the boys, on the other hand, we observed clusters of relatively larger GM volume in the hypothalamus and in the bilateral cerebellum, covering primarily the superior posterior lobes of the cerebellar hemispheres.
Regarding the comparisons involving the GD groups, we first compared the adolescents with GD to individuals sharing their gender identity. In comparison to natal boys, femaleto-male adolescents were found to have relatively smaller volumes in a cluster extending from the left fusiform gyrus to the superior posterior cerebellum. When comparing MF to natal girls, we observed smaller GM volumes in the right superior frontal and superior medial frontal cortex, and enlarged left cerebellar volume. The results of these comparisons are indicated in Table 2 and the whole-brain results are depicted in Fig. 1.

Next, we compared the GD groups to individuals sharing their natal sex. For these comparisons, no supra-threshold clusters surfaced at whole-brain level, suggesting that overall the regional GM volumes of MF and FM are largely in accordance with those observed in their natal sex. To further evaluate the degree of masculinization or feminization of $G M$ in individuals with GD, we compared GM volumes specifically within sexually dimorphic regions, by applying Regions of Interest (ROIs) representing the previously described regions of sex-typical volume (the ROls of larger volume in females to the contrasts ' $M F>M$ ' and ' $F>F M$ ' and those of larger male volume to ' $M>M F$ ' and ' $F M>F$ )'.

For these comparisons, we detected less volume in the right superior medial frontal cortex and more volume in the right cerebellum in $F M$ relative to girls without GD (see Table 3). Regarding MF, when examining GM volumes 

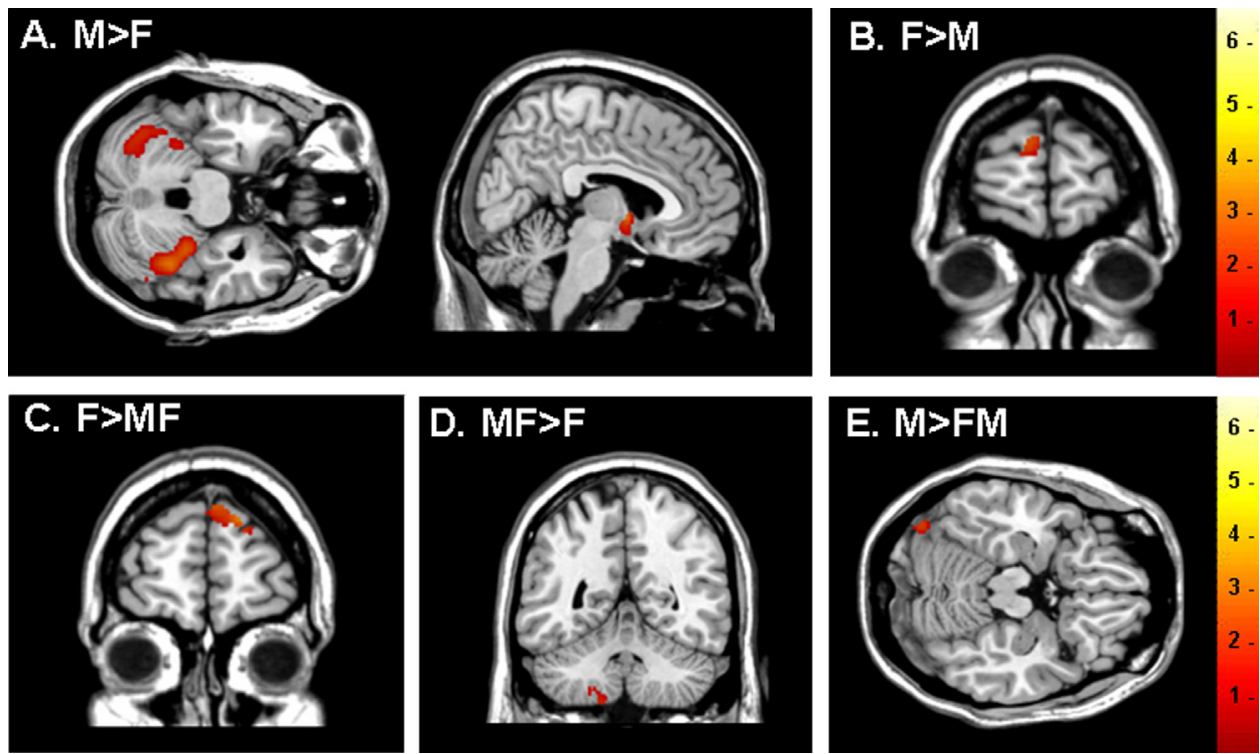

Fig. 1 Whole-brain results. Illustration of all the whole-brain results (see Table 2). For illustrative purposes, this figure displays the results at $p<0.0001$ (instead of $p<0.05 \mathrm{FWE}$-corrected) to make them stand out more from the background in the figure. $M=$ males without $G D ; F=$ females without $G D ; M F=$ males-to-female individuals with $G D ; F M=$ females-to-male individuals with $G D$.

Table 3 ROI results. Full results of the Marsbar ROI analyses comparing the GD groups and their respective natal sex.

\begin{tabular}{|c|c|c|c|}
\hline & Region & $T$ & Corrected $p$ \\
\hline \multicolumn{4}{|c|}{ Complete samples } \\
\hline \multirow[t]{3}{*}{$F M>F$} & L Cerebellum & 1.81 & 0.1014 \\
\hline & R Cerebellum ** & 3.86 & 0.0002 \\
\hline & Hypothalamus & 1.14 & 0.3364 \\
\hline$F>F M$ & $\begin{array}{l}\text { L superior } \\
\text { medial frontal } \\
\text { cortex }^{* *}\end{array}$ & 2.07 & 0.0198 \\
\hline$M F>M$ & $\begin{array}{l}\text { L superior } \\
\text { medial frontal } \\
\text { cortex }\end{array}$ & 1.43 & 0.0776 \\
\hline \multirow[t]{3}{*}{$M>M F$} & L Cerebellum ${ }^{* *}$ & 2.75 & 0.0033 \\
\hline & R Cerebellum ** & 2.98 & 0.0017 \\
\hline & Hypothalamus ${ }^{* *}$ & 2.40 & 0.0088 \\
\hline \multicolumn{4}{|c|}{ Untreated samples } \\
\hline \multirow[t]{3}{*}{$\mathrm{FM}>\mathrm{F}$} & L Cerebellum & 0.67 & 0.5850 \\
\hline & R Cerebellum ** & 2.63 & 0.0172 \\
\hline & Hypothalamus & 0.09 & 0.8986 \\
\hline$F>F M$ & $\begin{array}{l}\text { L superior } \\
\text { medial frontal } \\
\text { cortex }^{* *}\end{array}$ & 2.10 & 0.0205 \\
\hline$M F>M$ & $\begin{array}{l}\text { L superior } \\
\text { medial frontal } \\
\text { cortex }\end{array}$ & 0.92 & 0.8180 \\
\hline \multirow[t]{3}{*}{$M>M F$} & L Cerebellum ** & 3.15 & 0.0059 \\
\hline & R Cerebellum & 0.47 & 0.6851 \\
\hline & Hypothalamus* & 2.03 & 0.0765 \\
\hline
\end{tabular}

within these sexually dimorphic regions, we observed a trend for increased left superior medial frontal volume and significantly less volume in the bilateral superior posterior cerebellum and hypothalamus in comparison to boys without GD.

As a supplementary analysis, we also volumetrically compared the two GD groups to the individuals without GD in order to assess whether there were any alterations in GM volume accompanying GD (independent of sex), but no significant results surfaced for this comparison.

\subsubsection{Untreated sample}

To examine whether these deviations from the natal sex are not driven by exposure to the hormone therapy already provided to a subsample of our GD participants, we also repeated these comparisons including only GD adolescents who had not yet undergone any hormonal treatment at the time of the MRI acquisition and age-matched controls of their natal sex. To minimize the contribution of 'activational' effects of puberty-related sex steroid hormones, the stage of pubertal development was also included in these models as a nuisance covariate as an indication of the degree to which the body had already been exposed to pubertyrelated sex steroid hormones.

In line with the findings in the complete sample, we observed no deviations from the natal sex in either the FM or MF at whole-brain level ( $p<0.05$ FWE-corrected). However, when examining GM volumes within sexually dimorphic brain structures using ROI analyses, we observed differences in both GD groups with respect to their natal sex. More specifically, in FM, less GM volume was observed in the left superior medial frontal cortex in comparison to adolescent girls, while more GM volume was found in the right cerebellum than girls without GD. Regarding MF, in comparison to natal boys without GD, we observed smaller volumes in the 
left superior posterior hemisphere of the cerebellum and a trend for less volume in the hypothalamus.

We also compared the untreated FM and MF to control subjects sharing their gender identity rather than their natal sex. In accordance with the results including the complete sample, whereas no deviations in GM volumes were observed at whole-brain level ( $p<0.05$ FWE-corrected), when comparing both GD groups to adolescents sharing their gender identity we did observe volumetric differences with the natal sex at this whole-brain threshold ( $\mathrm{F}>\mathrm{MF}$ : Right inferior orbitofrontal cortex: 51 44-14, $T=6.80, p=0.006$ FWEcorrected. MF > F: - . M > FM: Right insula: $30237, T=5.87$, $p=0.006$ FWE-corrected. FM $>M$ : - ).

To examine the effects of hormone therapy on neural GM, we also directly compared: (a) the untreated $F M$ to $F M$ undergoing testosterone treatment, and (b) the untreated MF to MF exposed to estradiol treatment. For these analyses, no results were observed at whole-brain level. To further examine whether there were no differences between the groups within regions characterized by sex differences in the adolescent brain, we also applied the ROls representing sexually dimorphic brain structures to these comparisons. For FM, there was no significant difference in GM volume between untreated and crosssex-hormone-treated adolescents (treated $F M>$ untreated FM: Left cerebellum: $T=1.13$, corrected $p=0.346$. Right cerebellum: $T=1.03$, corrected $p=0.397$. Hypothalamus: $T=1.75$, corrected $p=0.127$. untreated $F M>$ treated $F M$ : Left medial frontal cortex: $T=0.08$, corrected $p=0.468$ ). Regarding the MF, we observed differences between the cross-sex hormone-treated sample and the untreated sample only in the left superior medial frontal cortex (treated MF > untreated MF: Left medial frontal cortex: $T=2.96$, corrected $p=0.004$. untreated MF $>$ treated MF: Left cerebellum: $T=0.14$, corrected $p=0.830$. Right cerebellum: $T=0.22$, corrected $p=0.800$. Hypothalamus: $T=0.43$, corrected $p=0.705)$.

\subsection{Multivariate analyses}

\subsubsection{Complete sample}

To examine whether GD individuals can be discriminated from natal boys and girls without GD based on spatially distributed patterns of GM volume, we applied a multivariate pattern classification analysis, incorporating a support vector machine algorithm. When using this approach for the classification of boys and girls without GD, we obtained a highly significant prediction accuracy of $88 \%$ for the discrimination between the sexes (see Table 4).

Next, we applied this approach to the GD groups and the adolescents sharing their gender identity. We obtained a high accuracy both for the classification of the FM and natal boys without GD and for the classification of the MF and girls without GD, showing that these GD groups can be discriminated from groups sharing their gender identity based on spatial GM patterns with a high accuracy (see Table 4).

For the classifications involving the GD groups and their respective natal sex, much lower prediction accuracy values were obtained. Only for the MF did this classification reach significance. This group could thus be distinguished from natal males slightly above chance level based on GM
Table 4 Multivariate results. Prediction accuracy values and $p$ values for the classifiers obtained using a multivariate pattern recognition approach.

\begin{tabular}{|c|c|c|c|}
\hline \multicolumn{2}{|l|}{ Class labels } & \multirow[t]{2}{*}{$\begin{array}{l}\text { Balanced } \\
\text { accuracy (\%) }\end{array}$} & \multirow[t]{2}{*}{$p$ Value } \\
\hline Group 1 & Group 2 & & \\
\hline$M$ & $\mathrm{~F}$ & 88.0 & $<0.0001$ \\
\hline \multirow[t]{2}{*}{ MF } & $M$ & 65.5 & 0.007 \\
\hline & $\mathrm{F}$ & 85.6 & $<0.0001$ \\
\hline \multirow{2}{*}{ Untreated MF } & $M$ & 45.5 & 0.61 \\
\hline & $F$ & 86.4 & $<0.0001$ \\
\hline \multirow[t]{2}{*}{$F M$} & $\mathrm{~F}$ & 52.2 & 0.32 \\
\hline & M & 78.4 & 0.002 \\
\hline \multirow[t]{2}{*}{ Untreated FM } & $\mathrm{F}$ & 50.0 & 0.55 \\
\hline & M & 76.5 & 0.006 \\
\hline
\end{tabular}

anatomy. The FM could not be discriminated from natal girls with a statistically significant accuracy.

For both GD groups, however, the classification accuracies for distinguishing them from individuals sharing their gender identity strongly exceed those for the discrimination from their natal sex based on spatially distributed GM. Fig. 2 displays the weight maps and confusion matrixes for each of these classifications.

Although a multivariate approach does not lend itself to extracting peak voxels based on the weight distribution, a visual inspection of the weight maps, which depict the relative contribution of each voxel to the classifier (see Fig. 2), suggests that voxels with a high relative weight are centered on the sexually dimorphic regions previously observed in the main model. To further investigate the contribution of these regions, we applied the ROls of the previously defined sexually dimorphic regions as a mask to replace the whole-brain mask commonly used for the analysis. Hence, the analyses involving the GD individuals and their natal sex were repeated using a mask of the sexually dimorphic structures. Using this mask, both GD groups could be discriminated from their natal sex with a significant prediction accuracy (FM-F: balanced accuracy: 47.7\%, $p=0.54$. MF-M: balanced accuracy: $61.7 \%, p=0.042$ ).

\subsubsection{Untreated sample}

We repeated these multivariate comparisons including only adolescents with GD who had not been exposed to any hormonal therapy at the time of the MRI acquisition and age-matched control subjects sharing either their natal sex or their gender identity. In line with the results obtained for the complete sample, we obtained a high accuracy for the classification of FM and natal boys without GD as well as for the classification of MF and natal girls without GD. Both GD groups could be significantly discriminated from adolescents sharing their gender identity based on the neural distribution of GM volume (see Table 4). 


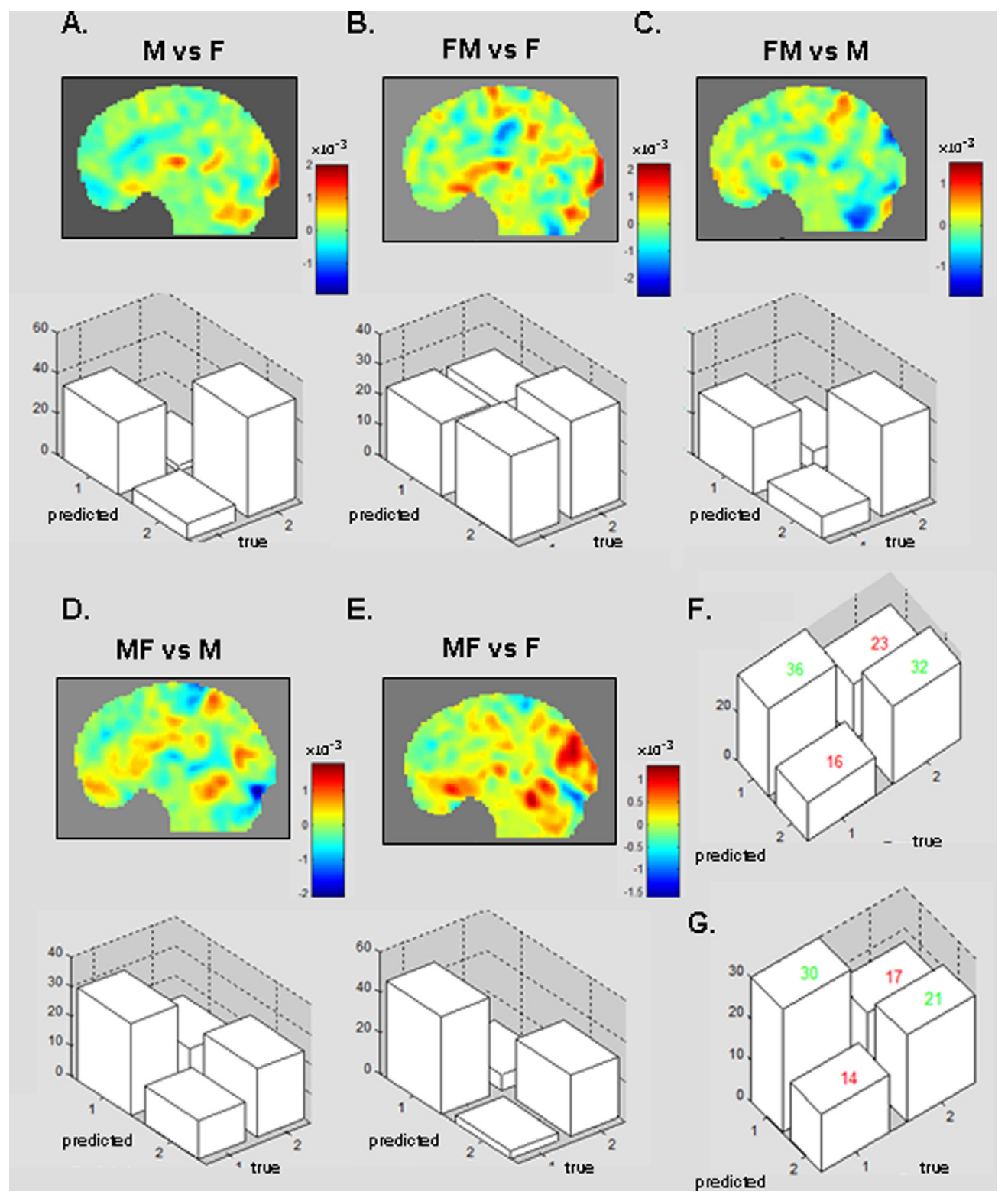

Fig. 2 Multivariate results. Confusion matrices and weight maps for the classification between (A) natal boys and girls, (B) FM and natal girls without GD, (C) FM and natal boys without GD, (D) MF and natal boys without GD, and (E) MF and natal girls without GD. Panels $F$ and $G$ display the confusion matrices for the FM versus girls and the MF versus boys respectively when using a mask of the sexually dimorphic structures. $M=$ males without GD; $F=$ females without GD; $M F$ = males-to-female individuals with GD; $F M=$ females-to-male individuals with $G D$.

In accordance with the results for the complete sample, much lower prediction accuracy values were obtained for the classification between the GD groups and their respective natal sex and the classification accuracies for discriminating MF or FM from individuals sharing their gender identity strongly exceed those for the classification from their natal sex. Neither the FM nor the MF could be discriminated from their natal sex with a statistically significant accuracy (see Table 4).

For completeness, we also repeated these multivariate analyses using the masks of the ROls of sexually dimorphic regions (as described above). When using this mask, the FM could still not significantly be discriminated from their natal sex, but a significant prediction accuracy was found for the classification between the untreated MF and boys without GD (FM-F: balanced accuracy: $50.0 \%, p=0.442$. MF-M: balanced accuracy: $77.3 \%, p=0.016)$.

\section{Discussion}

When comparing GM volume between adolescent boys and girls to identify sexually dimorphic brain structures, we observed clusters of enlarged GM volume in the hypothalamus and both superior posterior lobes of the cerebellar hemispheres in boys, while girls had more GM volume in the left superior medial frontal cortex. These clusters are centered on well-established regions of sexual dimorphism in the human brain. 
The hypothalamus, a structure richly endowed with sex steroid receptors, plays an essential role in reproduction and sexual behavior, and is a primary site of neural sex differences in animals (Dohler et al., 1982; Gorski et al., 1980; Matsumoto and Arai, 1983). In humans, postmortem studies have also discovered several sexually dimorphic nuclei with higher cell numbers and larger volume in males within this region (Allen et al., 1989; Swaab and Fliers, 1985). Clusters of enlarged hypothalamic volume in men as well as in adolescent boys have also been detected in vivo (Goldstein et al., 2001; Lombardo et al., 2012).

Like the hypothalamus, the cerebellum also represents a site of sex differences in the animal and human brain. It is well established that adult men have larger absolute and relative cerebellar volumes than women (Chung et al., 2005; Escalona et al., 1991; Filipek et al., 1994; Good et al., 2001; Raz et al., 2001; Tiemeier et al., 2010). In accordance with these volumetric differences, men were found to have a higher number of Purkinje cells than women (Hall et al., 1975). In children and adolescents, cerebellar volume is between 10 to $13 \%$ larger in boys than girls (Tiemeier et al., 2010). Interestingly, the superior posterior lobe of the cerebellum, which was bilaterally enlarged in adolescent boys in comparison to girls in our study, represents a site of especially robust sex differences in volumetric properties and developmental trajectory across adolescence (Tiemeier et al., 2010).

The region of larger GM volume in girls was located in the frontal cortex, which harbors some of the primary sexual dimorphisms of this type. Previous studies investigating sex differences in the human brain have also detected increased volume or cortical thickness in the GM comprising the superior frontal and more medial sections of the frontal cortex in women compared to men (Goldstein et al., 2001; Good et al., 2001; Lombardo et al., 2012; Luders et al., 2006, 2009a). It should be noted that in this study we did not obtain information regarding the menstrual cycle in the girls, and therefore we cannot exclude subtle cycle-related effects on GM volumes.

In line with theories of sexual differentiation, all the regions of sexual dimorphism identified in our study represent brain structures known to express high levels of sex steroid receptors during early brain development in other animal species (Goldstein et al., 2001), suggesting that brain regions homologous with those identified by animal studies as having a high sex steroid receptor density during critical periods of brain development are more likely to retain sexual dimorphisms throughout life. Accordingly, the volumetric differences observed between the GD groups and the control groups are also exclusively centered on brain structures rich in sex steroid receptors during fetal and early postnatal development.

In the GD groups, when examining the complete samples - i.e. the samples including GD individuals at different stages of treatment - we observed regional GM volumes and volumetric patterns of GM that were concordant with their natal sex. Volumetric differences were found between individuals with GD and adolescents sharing their gender identity in several regions across the brain, centered on sexually dimorphic structures. However, when comparing the GD groups to boys and girls of their natal sex, no differences were observed in local GM volumes at whole-brain level for either FM or MF.

Furthermore, multivariate pattern recognition analyses showed that for both GD groups the accuracy for distinguishing them from adolescents sharing their gender identity based on spatially distributed GM patterns was greater and more statistically significant than the accuracy for separating them from their natal sex. Thus at whole-brain level no signs of sex-atypical cerebral development were observed. However, when specifically examining sexually dimorphic structures in GD participants using ROI analyses, in both male-to-female and female-to-male adolescents we detected subtle deviations in GM volume from their respective natal sex in the direction of individuals sharing their gender identity. More specifically, FM had more volume in the right cerebellum and less volume in the left superior medial frontal cortex in comparison to girls without GD, while MF had less volume in the bilateral cerebellum and hypothalamus and a trend for more medial frontal volume than boys without GD.

When separately examining the untreated sample of MF and FM, a similar pattern was observed. At whole-brain level $(p<0.05$ FWE-corrected), differences in GM volume were only found in both GD groups with respect to subjects sharing their respective gender identity, but not in comparison to their natal sex. Likewise, using multivariate analyses, GD adolescents could only be discriminated from individuals sharing their gender identity based on spatially distributed neural GM patterns with a significant accuracy.

The ROI analyses separately examining the subsamples of GD adolescents who had not yet been exposed to any hormonal treatment also rendered similar results to those obtained using the complete GD samples, showing subtle deviations in GM volume from the natal sex within several of the sexually dimorphic structures in the direction of individuals sharing their gender identity. While untreated FM had less GM volume in the left superior medial frontal cortex but more volume in the right cerebellum in comparison to natal girls without GD, MF were found to have smaller volumes in the right cerebellar hemisphere and a trend for less volume in the hypothalamus compared to natal boys without GD.

In line with these findings, multivariate pattern classification analyses demonstrated that both GD groups could be successfully discriminated from their natal sex based on the cerebral GM volume distribution when taking into account only sexually dimorphic parts of the brain. In the untreated samples, a statistically significant prediction accuracy was only obtained for the classification between the MF and their natal sex when applying a mask of the sexually dimorphic structures.

The previous studies evaluating GM in adults with GD have rendered mixed results, some arguing against and some in support of a sex-atypical differentiation of the brain. One factor that likely contributes to this discrepancy is clinical heterogeneity. According to the Blanchard typology, there are two fundamentally different types of transsexualism (Blanchard et al., 1987). In the group commonly referred to as 'homosexual' or 'early-onset' individuals with GD, the sexual orientation is directed toward the natal sex, symptoms usually start early in childhood ('early onset') and physical differences have been observed with respect to individuals sharing their natal sex (e.g. MF were found 
to be shorter and lighter than men without GD (Blanchard et al., 1995), although this could not be replicated by other groups (Smith et al., 2005)). 'Non-homosexual' or 'late-onset' transsexuals, on the other hand, show a more variable sexual orientation (for instance directed at the other natal sex or asexual) and symptoms usually start during or after adolescence. In the present study, we investigated 'homosexual' /'early-onset' individuals with GD. Moreover, only individuals who arrived at the clinic at an early age and were followed at the center for many years were included in this study, allowing us to verify their 'early-onset' status rather than rely on retrospective questionnaires. This setup ensured a very reliable sample of 'early-onset' individuals with GD.

Savic and Arver (2011) examined GM in 'non-homosexual' MF using VBM and volumetric delineation and did not observe any signs of feminization, and Luders et al. (2009b) detected only a localized feminization of the right putamen in primarily 'non-homosexual' MF. Luders et al. (2012) also examined cortical thickness in MF, observing some deviations from the natal sex in several regions, although the lack of a female control sample did not facilitate determining whether these deviations reflect signs of feminization. On the other hand, Zubiaurre-Elorza et al. (2012) and Simon et al. (2013) investigated GM in 'homosexual' participants with GD. Simon et al. (2013) found that, in several brain regions, GD individuals differed from controls sharing their natal sex but not from those sharing their gender identity. Zubiaurre-Elorza et al. (2012) performed the first study investigating GM in 'homosexual'/'early-onset' participants with GD. They observed differences in volume of the putamen between FM and women without GD while cortical thickness did not differ between these groups. MF, on the other hand, deviated from men without GD in cortical thickness in several areas across the brain but not from women. This lead them to conclude that signs of subcortical masculinization could be observed in FM while MF exhibited signs of feminization in the cerebral cortex (Zubiaurre-Elorza et al., 2012).

Considering these findings, it is interesting to note that, in our study, when searching for sexually dimorphic structures in natal boys and girls without GD, we observed subcortical regions of male-dominant volume, while the locus of female-biased volume was located within the cerebral mantle. These findings suggest that there is a distinction between the degree to which feminization and masculinization processes affect subcortical and cortical sections of the brain, although in the male-to-female and female-tomale groups we did not observe a selective predilection for cortical versus subcortical alterations, respectively.

The sex-atypical differentiation of the brain integral to most theories on the pathogenesis of GD is commonly postulated to reflect exposure to an altered neuroendocrine climate in utero. However, besides the early organizational effects of gonadal hormones, exposure to sex steroids later in life, e.g. via puberty-related endogenous hormone surges or cross-sex hormone therapy, can exert activational effects on the central nervous system. Although puberty is a very interesting period to study the brain as pubertal sex steroid hormones are exerting activational effects on the central nervous system, it is also a period characterized by a high variability in the degree of pubertal maturation. In our study, we also examined GM volumes in the subsamples of GD adolescents who had not yet been exposed to any form of hormone therapy, and we observed gender-atypical volumes in several of the sexually dimorphic structures. In order to further minimize the contribution of activational effects of sex steroid hormones during adolescence and enhance the focus on organizational effects, we also controlled for the stage of pubertal development in these analyses, taken as an index of the body's degree of exposure to endogenous puberty-related sex steroids. Our results suggest that, before the manifestation of contingent neuroanatomical changes induced by endogenous puberty-related sex steroid surges or treatment with exogenous cross-sex steroid hormones, there are already subtle deviations from the natal sex in individuals with GD, which may reflect signs of a partial sex-atypical brain differentiation during early development. The fact that the analyses involving the complete samples and those involving the untreated samples point in the same direction and that no or very little differences were observed between the treated and untreated samples also point to organizational rather than activational effects.

However, although the localization of the significant clusters in brain structures expressing high levels of sex steroid receptors during fetal development carefully points to an endocrine effect, the current study does not allow determining whether these deviations reflect endocrine, environmental (e.g. differences in upbringing, interest or activities) or genetic influences or a combination of these factors. It should also be noted that the sample sizes of the GD individuals who had never been exposed to any hormonal treatment are quite small $(N=17$ for the FM and $N=11$ for the MF). Therefore, these findings should be interpreted with caution.

Another limitation of the present study is that it does not provide the ideal framework for investigating the effects of cross-sex hormone therapy on brain structure due to its cross-sectional setup. We also did not obtain sex steroid hormone levels at the time of the MRI acquisitions, which might have been used for correlation analyses. A few previous studies have examined this topic in individuals with GD using a sensitive longitudinal approach. Interestingly, these studies indicated that androgen treatment in FM rendered increases in total brain volume, regional cortical thickness and hypothalamic volume, while estrogen therapy accompanied by anti-androgen treatment in MF was associated with reductions in total brain volume, cortical thickness and subcortical gray matter volume (Hulshoff Pol et al., 2006; Zubiaurre-Elorza et al., 2014). These studies suggest that, although sexually dimorphic structures seem to have already partially been programmed in a sex-atypical manner in GD children who have not yet been exposed to cross-sex hormone therapy, sex steroid hormones administered as part of cross-sex hormone therapy in adulthood can further sculpt the brain into a morphological configuration that fits the individual's gender identity rather than the natal sex.

In sum, sexual dimorphisms in GM volume were observed in several structures of the adolescent brain known to express high levels of sex steroid receptors during fetal and early postnatal development in rodents. Regarding the comparisons involving the GD groups, at whole-brain level they differed from adolescents sharing their gender identity while there were no deviations in GM volume with respect to the natal sex, arguing against a sex-atypical 
differentiation of the brain in gender dysphoria. Likewise, multivariate pattern recognition analyses showed that classification accuracies for discriminating the GD groups from individuals sharing their gender identity exceed the accuracies obtained for the classification between the GD adolescents and their natal sex.

However, when examining GM volumes within sexually dimorphic structures using ROI analyses, within both GD groups we also observed subtle deviations from the natal sex where GM volumes diverged from the natal sex toward the groups sharing their gender identity. Multivariate analyses also demonstrated that the individuals with GD could significantly be discriminated from their natal sex when taking into account only the GM distribution within sexually dimorphic structures.

Similar results were observed in the subsamples of untreated FM and MF who had not been exposed to any form of hormonal therapy - while also controlling for the stage of pubertal development - within several of these regions, suggesting that the GD brain may have already partially been programmed in a sex-atypical manner during early development.

Taken together, the results of this study indicate that the neuroanatomical characteristics of adolescents with GD are primarily concordant with individuals of their respective natal sex. However, within sexually dimorphic brain structures we observed subtle signs of a volumetric divergence from the natal sex in the direction of individuals sharing their gender identity, which may point to a partial sex-atypical differentiation of the brain.

\section{Role of the funding source}

The funding sources did not play a role in any component of this study.

\section{Conflict of interest statement}

None declared.

\section{Acknowledgements}

We would like to thank all the participants and their parents. This work was supported by an educational grant from Ferring BV, Hoofddorp, and by a $\mathrm{VICI}$ grant (453-08-003) from the Dutch Science Foundation (Nederlandse Organisatie voor Wetenschappelijk Onderzoek) to JB. JB is a senior research associate of the Belgian Fonds National de la Recherche Scientifique.

\section{References}

Achenbach, T.M., Edelbrock, C.S., 1983. Manual for the Child Behavior Checklist and Revised Child Behavior Profile. University of Vermong, Department of Psychiatry, Burlington, VT.

Allen, L.S., Hines, M., Shryne, J.E., Gorski, R.A., 1989. Two sexually dimorphic cell groups in the human brain. J. Neurosci. 9 , 497-506.

American Psychiatric Association, 2000. Diagnostic and Statistical Manual of Mental Health Disorders (Revised 4th ed.). Author, Washington, DC.
Arnold, A.P., 2004. Sex chromosomes and brain gender. Nat.Rev Neurosci. 5, 701-708.

Ashburner, J., Friston, K.J., 2000. Voxel-based morphometry-the methods. Neurolmage 11, 805-821.

Bakker, J., Baum, M.J., 2008. Role for estradiol in female-typical brain and behavioral sexual differentiation. Front. Neuroendocrinol. 29, 1-16.

Bakker, J., De Mees, C., Douhard, Q., Balthazart, J., Gabant, P., Szpirer, J., Szpirer, C., 2006. Alpha-fetoprotein protects the developing female mouse brain from masculinization and defeminization by estrogens. Nat. Neurosci. 9, 220-226.

Bao, A.M., Swaab, D.F., 2011. Sexual differentiation of the human brain: relation to gender identity, sexual orientation and neuropsychiatric disorders. Front. Neuroendocrinol. 32, 214-226.

Blanchard, R., Clemmensen, L.H., Steiner, B.W., 1987. Heterosexual and homosexual gender dysphoria. Arch. Sex. Behav. 16, 139-152.

Blanchard, R., Dickey, R., Jones, C.L., 1995. Comparison of height and weight in homosexual versus nonhomosexual male gender dysphorics. Arch. Sex. Behav. 24, 543-554.

Carruth, L.L., Reisert, I., Arnold, A.P., 2002. Sex chromosome genes directly affect brain sexual differentiation. Nat. Neurosci. 5, 933-934.

Chung, S.C., Lee, B.Y., Tack, G.R., Lee, S.Y., Eom, J.S., Sohn, J.H., 2005. Effects of age, gender, and weight on the cerebellar volume of Korean people. Brain Res. 1042, 233-235.

Cohen-Kettenis, P.T., Gooren, L.J., 1999. Transsexualism: a review of etiology, diagnosis and treatment. J. Psychosom. Res. 46, 315-333.

Coleman, E., Bockting, W., Botzer, M., Cohen-Kettenis, P., DeCuypere, G., Feldman, J., Fraser, L., Green, J., Knudson, G., Meyer, W.J., Monstrey, S., Adler, R.K., Brown, G.R., Devor, A.H., Meyer-Bahlburg, H., Hall, B.P., Pfaefflin, F., Rachlin, K., Robinson, B., Schechter, L.S., Tangpricha, V., van Trotsenburg, M., Vitale, A., Winter, S., Whittle, S., Wylie, K.R., Zucker, K., 2011. Standards of care for the health of transsexual, transgender, and gender nonconforming people, 7 th version. Int. J. Transgend. 13, 165-232.

Dohler, K.D., Coquelin, A., Davis, F., Hines, M., Shryne, J.E., Gorski, R.A., 1982. Differentiation of the sexually dimorphic nucleus in the preoptic area of the rat brain is determined by the perinatal hormone environment. Neurosci. Lett. 33, 295-298.

Dorn, L.D., 2006. Measuring puberty. J. Adolesc. Health 39, 625-626.

Escalona, P.R., McDonald, W.M., Doraiswamy, P.M., Boyko, O.B., Husain, M.M., Figiel, G.S., Laskowitz, D., Ellinwood Jr., E.H., Krishnan, K.R., 1991. In vivo stereological assessment of human cerebellar volume: effects of gender and age. AJNR Am. J. Neuroradiol. 12, 927-929.

Filipek, P.A., Richelme, C., Kennedy, D.N., Caviness Jr., V.S., 1994. The young adult human brain: an MRI-based morphometric analysis. Cereb. Cortex 4, 344-360.

Garcia-Falgueras, A., Swaab, D.F., 2008. A sex difference in the hypothalamic uncinate nucleus: relationship to gender identity. Brain 131, 3132-3146.

Goldstein, J.M., Seidman, L.J., Horton, N.J., Makris, N., Kennedy, D.N., Caviness Jr., V.S., Faraone, S.V., Tsuang, M.T., 2001. Normal sexual dimorphism of the adult human brain assessed by in vivo magnetic resonance imaging. Cereb. Cortex 11, 490-497.

Good, C.D., Johnsrude, I., Ashburner, J., Henson, R.N., Friston, K.J., Frackowiak, R.S., 2001. Cerebral asymmetry and the effects of sex and handedness on brain structure: a voxel-based morphometric analysis of 465 normal adult human brains. Neurolmage 14, 685-700.

Gorski, R.A., Harlan, R.E., Jacobson, C.D., Shryne, J.E., Southam, A.M., 1980. Evidence for the existence of a sexually dimorphic nucleus in the preoptic area of the rat. J. Comp. Neurol. 193, 529-539. 
Hall, T.C., Miller, A.K.H., Corsellis, J.A.N., 1975. Variations in the human Purkinje cell population according to sex and age. Neuropathol. Appl. Neurobiol. 1, 267-292.

Hembree, W.C., Cohen-Kettenis, P., Delemarre-van de Waal, H.A., Gooren, L.J., Meyer III, W.J., Spack, N.P., Tangpricha, V., Montori, V.M., 2009. Endocrine treatment of transsexual persons: an Endocrine Society clinical practice guideline. J. Clin. Endocrinol. Metab. 94, 3132-3154.

Heylens, G., De Cuypere, G., Zucker, K.J., Schelfaut, C., Elaut, E., Vanden Bossche, B.H., De Baere, E., T'Sjoen, G., 2012. Gender identity disorder in twins: a review of the case report literature. J. Sex Med. 9, 751-757.

Hines, M., Ahmed, S.F., Hughes, I.A., 2003. Psychological outcomes and gender-related development in complete androgen insensitivity syndrome. Arch. Sex. Behav. 32, 93-101.

Hulshoff Pol, H.E., Cohen-Kettenis, P.T., Van Haren, N.E.M., Peper, J.S., Brans, R.G.H., Cahn, W., Schnack, H.G., Gooren, L.J.G., Kahn, R.S., 2006. Changing your sex changes your brain: influences of testosterone and estrogen on adult human brain structure. Eur. J. Endocrinol. 155, S107-S114.

Kreukels, B.P., Cohen-Kettenis, P.T., 2011. Puberty suppression in gender identity disorder: the Amsterdam experience. Nat. Rev. Endocrinol. 7, 466-472.

Kruijver, F.P., Zhou, J.N., Pool, C.W., Hofman, M.A., Gooren, L.J., Swaab, D.F., 2000. Male-to-female transsexuals have female neuron numbers in a limbic nucleus. J. Clin. Endocrinol. Metab. 85, 2034-2041.

Lombardo, M.V., Ashwin, E., Auyeung, B., Chakrabarti, B., Taylor, K., Hackett, G., Bullmore, E.T., Baron-Cohen, S., 2012. Fetal testosterone influences sexually dimorphic gray matter in the human brain. J. Neurosci. 32, 674-680.

Luders, E., Gaser, C., Narr, K.L., Toga, A.W., 2009a. Why sex matters: brain size independent differences in gray matter distributions between men and women. J. Neurosci. 29, 14265-14270.

Luders, E., Narr, K.L., Thompson, P.M., Rex, D.E., Woods, R.P., Deluca, H., Jancke, L., Toga, A.W., 2006. Gender effects on cortical thickness and the influence of scaling. Hum. Brain Mapp. 27, 314-324.

Luders, E., Sanchez, F.J., Gaser, C., Toga, A.W., Narr, K.L., Hamilton, L.S., Vilain, E., 2009b. Regional gray matter variation in male-to-female transsexualism. Neurolmage 46, 904-907.

Luders, E., Sanchez, F.J., Tosun, D., Shattuck, D.W., Gaser, C., Vilain, E., Toga, A.W., 2012. Increased cortical thickness in maleto-female transsexualism. J. Behav. Brain Sci. 2, 357-362.

Matsumoto, A., Arai, Y., 1983. Sex difference in volume of the ventromedial nucleus of the hypothalamus in the rat. Endocrinol. Jpn. 30, 277-280.

McCarthy, M.M., 2008. Estradiol and the developing brain. Physiol. Rev. 88, 91-124.

Rametti, G., Carrillo, B., Gomez-Gil, E., Junque, C., Segovia, S., Gomez, A., Guillamon, A., 2011a. White matter microstructure in female to male transsexuals before cross-sex hormonal treatment. A diffusion tensor imaging study. J. Psychiatr. Res. 45, 199-204.
Rametti, G., Carrillo, B., Gomez-Gil, E., Junque, C., ZubiarreElorza, L., Segovia, S., Gomez, A., Guillamon, A., 2011b. The microstructure of white matter in male to female transsexuals before cross-sex hormonal treatment. A DTI study. J. Psychiatr. Res. 45, 949-954.

Raz, N., Gunning-Dixon, F., Head, D., Williamson, A., Acker, J.D., 2001. Age and sex differences in the cerebellum and the ventral pons: a prospective MR study of healthy adults. AJNR Am. J. Neuroradiol. 22, 1161-1167.

Savic, I., Arver, S., 2011. Sex dimorphism of the brain in male-tofemale transsexuals. Cereb. Cortex 21, 2525-2533.

Savic, I., Garcia-Falgueras, A., Swaab, D.F., 2010. Sexual differentiation of the human brain in relation to gender identity and sexual orientation. Prog. Brain Res. 186, 41-62.

Schrouff, J., Rosa, M.J., Rondina, J.M., Marquand, A.F., Chu, C. Ashburner, J., Phillips, C., Richiardi, J., Mourao-Miranda, J., 2013. PRoNTo: pattern recognition for neuroimaging toolbox. Neuroinformatics 11, 319-337.

Simon, L., Kozak, L.R., Simon, V., Czobor, P., Unoka, Z., Szabo, A., Csukly, G., 2013. Regional grey matter structure differences between transsexuals and healthy controls-a voxel based morphometry study. PLoS ONE 8, e83947.

Smith, Y.L., van Goozen, S.H., Kuiper, A.J., Cohen-Kettenis, P.T., 2005. Sex reassignment: outcomes and predictors of treatment for adolescent and adult transsexuals. Psychol. Med. 35, 89-99.

Swaab, D.F., 2007. Sexual differentiation of the brain and behavior. Best Pract. Res. Clin. Endocrinol. Metab. 21, 431-444.

Swaab, D.F., Fliers, E., 1985. A sexually dimorphic nucleus in the human brain. Science 228, 1112-1115.

Tanner, J., 1962. Growth at Adolescence. Blackwell Scientific Publications; Oxford, UK.

Tiemeier, H., Lenroot, R.K., Greenstein, D.K., Tran, L., Pierson, R., Giedd, J.N., 2010. Cerebellum development during childhood and adolescence: a longitudinal morphometric MRI study. Neurolmage 49, 63-70.

Verhulst, F.C., van der Ende, J., Koot, H.M., 1996. Handleiding voor de CBCL-4/ 18 [Manual for the CBCL/4-18], Sophia Kinderziekenhuis, Erasmus MC; Rotterdam, the Netherlands.

Wechsler, D., 1997. Wechsler Adult Intelligence Scale-Third Edition. The Psychological Corporation, San Antonio, TX.

Wechsler, D., 1991. Wechsler Intelligence Scale for Children-Third Edition. The Psychological Corporation, San Antonio, TX.

Wisniewski, A.B., Migeon, C.J., Meyer-Bahlburg, H.F., Gearhart, J.P., Berkovitz, G.D., Brown, T.R., Money, J., 2000. Complete androgen insensitivity syndrome: long-term medical, surgical, and psychosexual outcome. J. Clin. Endocrinol. Metab. 85, 2664-2669.

Zhou, J.N., Hofman, M.A., Gooren, L.J., Swaab, D.F., 1995. A sex difference in the human brain and its relation to transsexuality. Nature 378, 68-70.

Zubiaurre-Elorza, L., Junque, C., Gomez-Gil, E., Guillamon, A., 2014. Effects of cross-sex hormone treatment on cortical thickness in transsexual individuals. J. Sex. Med. 11, 1248-1261.

Zubiaurre-Elorza, L., Junque, C., Gomez-Gil, E., Segovia, S., Carrillo, B., Rametti, G., Guillamon, A., 2012. Cortical Thickness in Untreated Transsexuals. Cereb. Cortex 23 (12), 2855-2862. 